\title{
Hypo-methylation mediates chromosomal instability in pancreatic NET
}

\author{
I Marinoni1, A Wiederkeher1, T Wiedmer1,2, S Pantasis', A Di Domenico1,2, R Frank', \\ E Vassella', A Schmitt1 and A Perren'1
}

IInstitute of Pathology, University of Bern, Bern, Switzerland

2GCB Graduate School Bern, Bern, Switzerland
Correspondence should be addressed to I Marinoni Email

Ilaria.marinoni@pathology. unibe.ch

\begin{abstract}
DAXX and or ATRX loss occur in $40 \%$ of pancreatic neuroendocrine tumors (PanNETs). PanNETs negative for DAXX or ATRX show an increased risk of relapse. The tumorassociated pathways activated upon DAXX or ATRX loss and how this event may induce chromosomal instability (CIN) and alternative lengthening telomeres (ALT) are still unknown. Both DAXX and ATRX are involved in DNA methylation regulation. DNA methylation of heterochromatin and of non-coding sequences is extremely important for the maintenance of genomic stability. We analyzed the association of DAXX and/or ATRX loss and CIN with global DNA methylation in human PanNET samples and the effect of DAXX knock-down on methylation and cell proliferation. We assessed LINE1 as well as global DNA methylation in 167 PanNETs, and we found that DAXX and or ATRX-negative tumors and tumors with CIN were hypomethylated. DAXX knock-down in PanNET cell lines blocked cells in G1/G0 phase and seemed to increase CIN in QGP-1 cells. However, no direct changes in DNA methylation were observed after DAXX knock-down in vitro. In conclusion, our data indicate that epigenetic changes are crucial steps in the progression of PanNETs loss and suggest that DNA methylation is the mechanism via which CIN is induced, allowing clonal expansion and selection.
\end{abstract}

\author{
Key Words \\ - pancreatic neuroendocrine \\ tumors \\ - DNA methylation \\ - chromosomal instability \\ - DAXX and ATRX
}

Endocrine-Related Cancer (2017) 24, 137-146

\section{Introduction}

The molecular pathways and mechanisms underlying initiation and progression of pancreatic neuroendocrine tumors (PanNET) are still poorly understood. Mutations in DAXX (death domain-associated protein) and ATRX (ATR-X) with correspondent loss of protein expression in the tumor tissue occur in 40\% of PanNETs (Jiao et al. 2011). DAXX and or ATRX loss correlates with chromosomal instability (CIN) and predicts for relapse in low-stage patients (Stages I to III in the absence of distant metastasis) (Marinoni et al. 2014). DAXX- and or ATRX-negative tumors show alternative lengthening of telomeres (ALT), a telomerase-independent mechanism for telomere length maintenance, based on homologous recombination (Heaphy et al. 2011). The mechanisms and the sequence of events linking DAXX/ATRX mutation, ALT and CIN are unknown.

In addition to mutations, the importance of epigenetic changes is increasingly recognized for many cancer types (reviewed in Das and Singal (2004)). DAXX and ATRX participate in maintaining the epigenetic status of the cells by regulating both DNA methylation and H3.3 deposition at telomeric and peri-centromeric regions. DAXX interacts and recruits DNA methyl-transferase enzyme 1 (DNMT1) to specific promoters including

Published by Bioscientifica Ltd 
RASSF1 and RELB (Puto \& Reed 2008). ATRX contains the ADD domain as does DNMT3-DNMT3L, important for establishing and maintaining DNA methylation pattern (Noh et al. 2016).

Impairment of DNA methylation in PanNETs has been shown in several genes (VHL, cdkn2a (p16), RASSF1, DAPK1, TIMP3, PAX5, HIC1, CADM1, PYCARD, ESR1, VHL, RARB, WT1 and MGMT) (House et al. 2003, Schmitt et al. 2009, Malpeli et al. 2011, Schmitt et al. 2014, Stefanoli et al. 2014). Genome-wide methylation profiling of PanNET has shown a difference of methylation pattern between DAXX- and ATRX-negative tumors, suggesting that mainly DAXX loss is driving DNA methylation changes in PanNETs (Pipinikas et al. 2015).

DNA methylation regulates not only gene expression but also genomic stability. Indeed, heterochromatin and silenced regions are usually hyper-methylated resulting in a highly condensed chromatin structure not accessible to the transcription machinery. Hypo-methylated DNA regions are usually highly transcribed. Repetitive and mobile elements such as LINE1 (long interspersed element-1) and $A L U$ (Arthrobacter luteus) sequences are preserved from transcription and activation by DNA hyper-methylation. Once activated, these sequences move within the genome and thereby generate chromosomal instability. Hypo-methylation of LINE1 and $A L U$ sequences occurs in several tumors including PanNETs (Choi et al. 2007, Stefanoli et al. 2014). It is currently unknown whether LINE1 and ALU hypo-methylation is directly linked to $D A X X$ and or ATRX mutation. Regulation of transcription of the telomeric sequences TERRA (telomeric repeatcontaining RNA) and telomere stability is also dependent on the DNA methylation status. Dnmt1-deficient mouse embryonic stem cells show elongated telomeres, increased recombination at telomeric regions and ALT activation (Gonzalo et al. 2006).

In the present study, we aimed to analyze the effect of DAXX and or ATRX loss on global methylation in ex vivo human PanNETs samples and in vitro in BON-1 and QGP-1 cell line models. In vitro we also assessed the genomic instability induction upon DAXX knock-down. We found that DAXX- and/or ATRX-negative PanNETs showed global hypo-methylation but not LINE1 hypomethylation compared to positive tumors. Additionally, we were able to find a link between LINE1 hypomethylation and CIN in PanNETs. DAXX knock-down does not induce methylation changes in BON-1 and QGP-1 cells.

\section{Materials and methods}

\section{Human samples}

167 out of 207 resected well-differentiated PanNETs (G1-G2) and 11 matching controls were used in the study based on DNA viability and informative IHC results (Marinoni et al. 2014).

CIN data obtained by comparative genomic hybridization (CGH) were previously reported (Marinoni et al. 2014).

CIN were defined as showing a total number of gains and losses of 8 or more in conventional CGH and of 20 or more in array CGH. The use of patient material was approved by the local ethics committees (Number 105/2015). The composition of samples is described in Table 1 and in the Supplementary Fig. 1 (see section on supplementary data given at the end of this article).

\section{Cell line culture conditions and treatments}

The pancreatic neuroendocrine cell line BON-1 (pancreatic carcinoid) was provided by E.J. Speel, Department of Pathology, Maastricht, The Netherlands, and metastatic somatostatinoma-derived QGP-1 cell line was obtained from Japanese Health Sciences Foundation, Osaka, Japan. Both cell lines authentication was performed by STRs analysis (Microsynth, Balgach, Switzerland) upon arrival (2011) and in the present year. QGP-1 cells resulted in 100\% matching with the QGP-1 profile (GNE586559, Genentech, Roche, Basel, Switzerland). BON-1 cells are not commercially available; therefore, no comparison is possible; however, STRs analysis of these cells revealed no matching with any data available in the ATCC database, excluding any contamination with other cell lines. Additionally, the expression of the neuroendocrine markers synaptophysin and chromogranin-A was checked yearly. Both QGP-1 and BON-1 cells express the two markers. Cells were kept in culture for a maximum of 20-25 passages.

Both cell lines were cultured at $37^{\circ} \mathrm{C}$ under $5 \% \mathrm{CO}_{2}$. BON-1 was cultured in DMEM/Nutrient Mixture F-12 Ham (D6421, Sigma-Aldrich) and QGP-1 in RPMI-1640 Dutch modification (R7638, Sigma-Aldrich), both media supplemented with 10\% FBS (GIBCO), 2nM L-alanyl-Lglutamine (Sigma-Aldrich), $100 \mu \mathrm{g} / \mathrm{mL}$ streptomycin and 100 units/mL of penicillin (Sigma-Aldrich). 
Table 1 Characteristics of the human sample collective.

\begin{tabular}{|c|c|c|c|}
\hline Collective & $\begin{array}{c}\text { Total patients } \\
\text { (IHC plus LINE1 } \\
\text { and global } \\
\text { methylation) }\end{array}$ & IHC & $\begin{array}{c}\text { LINE1 and global } \\
\text { methylation }\end{array}$ \\
\hline Tot & 167 & 153 & 55 \\
\hline Female & 82 & 75 & 26 \\
\hline \multirow[t]{2}{*}{ Male } & 83 & 76 & 28 \\
\hline & 2 & 2 & 1 \\
\hline Age average & 56,66 & 56,09 & 55,21 \\
\hline G1 & 119 & 107 & 33 \\
\hline G2 & 48 & 46 & 22 \\
\hline T1 & 61 & 53 & 13 \\
\hline T2 & 46 & 44 & 18 \\
\hline T3-4 & 45 & 41 & 23 \\
\hline NA & 15 & 15 & 1 \\
\hline No & 58 & 54 & 15 \\
\hline N1 & 39 & 36 & 24 \\
\hline NA & 70 & 63 & 16 \\
\hline MO & 95 & 85 & 34 \\
\hline M1 & 30 & 30 & 12 \\
\hline NA & 42 & 38 & 9 \\
\hline DAXX/ATRX+ & 74 & 67 & 13 \\
\hline DAXX/ATRX- & 47 & 44 & 30 \\
\hline NA & 46 & 42 & 12 \\
\hline DAXX- & 23 & 23 & 3 \\
\hline $\mathrm{DAXX}+$ & 106 & 97 & 38 \\
\hline NA & 38 & 33 & 14 \\
\hline ATRX- & 28 & 25 & 13 \\
\hline ATRX+ & 95 & 88 & 33 \\
\hline NA & 44 & 40 & 9 \\
\hline ALT- & 59 & 53 & 16 \\
\hline ALT+ & 39 & 37 & 20 \\
\hline NA & 69 & 63 & 19 \\
\hline $\mathrm{CIN}-$ & 22 & 15 & 11 \\
\hline $\mathrm{CIN}+$ & 30 & 26 & 11 \\
\hline NA & 115 & 112 & 33 \\
\hline MEN1 wt & 15 & 15 & 15 \\
\hline MEN1 mut & 11 & 11 & 11 \\
\hline NA & 141 & 127 & 29 \\
\hline MGMT hyper & & & 8 \\
\hline \multirow[t]{2}{*}{ MGMT hypo } & & & 17 \\
\hline & & & 30 \\
\hline RFS (months) & & & $\begin{array}{c}52 \text { (data based on } \\
44 \text { patients) }\end{array}$ \\
\hline TSS (months) & & & $\begin{array}{c}80 \text { (data based on } \\
44 \text { patients) }\end{array}$ \\
\hline
\end{tabular}

G (grade), T (tumor stage), N (lymph node metastasis), M (distant metastasis), ALT (alternative lengthening telomeres), CIN (chromosomal instability), RFS (relapse-free survival), TSS (tumor-specific survival).

\section{Immunohistochemistry (IHC)}

Four-micrometer sections were taken from a TMA including 207 pNETs derived from patients who underwent surgery at the Inselspital Bern, Switzerland as previously described (Marinoni et al. 2014) and stained with an anti-5-methylcytidine antibody
BI_MECY_0100 (Eurogentec, Kaneka, Liege, Belgium). The Immunohistochemical staining was performed on an automated staining system (Leica Bond III; Leica Biosystems, Nunningen, Switzerland). Antigen retrieval was performed by heating citrate buffer at $100^{\circ} \mathrm{C}$ for $30 \mathrm{~min}$. The primary antibody was incubated for $30 \mathrm{~min}$ at a dilution of 1:200. Visualization was performed using the avidin-biotin complex method, which yielded a brown staining signal. Normal pancreatic islets show strong positive staining for 5-methylcytidine. We therefore scored samples which showed similar or stronger staining than normal islets as 'high-methylated', and samples that showed weaker staining than normal islets as 'low-methylated'. To exclude false-negative samples, only samples with positive nuclear staining of non-neoplastic cells and negative tumor nuclei were scored as negative (153 of 207 samples remaining informative). 54 samples with both negative tumor nuclei and non-neoplastic stromal and endothelial cells were scored as non-informative and excluded from further analysis.

\section{DNA extraction, bisulfite conversion and global methylation analysis}

DNA was extracted from cell pellet of about $6 \times 10^{5}$ cells or from paraffin-embedded human PanNETs (55 tumor samples with more than $80 \%$ tumor content and 11 matching normal pancreatic tissues, Table 1 and Supplementary Fig. 1) using Nucleo Spin kit by MachereyNagel (Düren, Germany) according to the manufacturer's instructions. For bisulfite conversion of gDNA, the EZ DNA Methylation-Gold kit by Zymo Research Corporation (Freiburg, Germany) was used following the manufacturer's instructions.

Genomic DNA of 10 tumor samples was analyzed by its global methylation status using EpiSeeker methylated DNA Quantification Kit (ab117128, Abcam), according to the supplier's instructions. Colorimetric measurements were done on an ELISA reader (Tecan, Männerdorf, Switzerland). Hyper-methylated and hypo-methylated DNAs (Zymo Research, Freiburg, Germany) were used as controls.

PCR and pyrosequencing investigation for LINE-1 for 51 samples (GenBank accession number X58075) methylation analysis was performed using PyroMark kit (Qiagen). To analyze sequence methylation of LINE-1, the PyroMark Q24 CpG LINE-1 $(4 \times 24)$ methylation detection assay was used (Qiagen). Amplifications were performed with Master Mix PyroMark PCR Kit (Qiagen) according to supplier's instructions on a Veriti System gradient apparatus (Applied Biosystems, 
Thermo Fisher Scientific). PCR product was bound to Streptavidin Sepharose HP (Amersham Biosciences), purified, washed, denatured and washed again with the PyroMark Q24 Vacuum Workstation 220V (Qiagen). Pyrosequencing was performed using the Qiagen PyroMark Q24 System with the PyroMark Q24 Cartridge Method 0011 (Qiagen). The assay setups and analysis were performed with PyroMark Assay Design Software, V.1.0.6 (Qiagen).

MGMT (O-6-methylguanine-DNA-methyltransferase) promoter methylation was assessed in 25 tumor samples as previously described (Vassella et al. 2011). Samples with methylation level higher and equal to $5 \%$ were considered hyper-methylated and samples with methylation level $<5 \%$ were considered hypo-methylated.

The MEN1 gene was analyzed in 26 tumor samples by semi-conductive sequencing using an Ion Torrent PGM (Life Technologies). Protein-coding exons were amplified by multiplex polymerase chain reaction using 2 primer pools designed by the Ion AmpliSeq Designer (Life Technologies). Library construction, emulsion polymerase chain reaction and sequencing were performed according to the manufacturer's recommendations. The Torrent Suite 5.0.3 platform was used for sequence alignment with the hg19 human genome reference. Variant calling was performed with the variant caller 5.0.3.5. and the IonReporter 5.0 software (Life Technologies). The average base coverage depth for most samples was more than 2000 reads. MEN1 mutations found are summarized in Supplementary Table 1 (see section on supplementary data given at the end of this article).

\section{DAXX knock-down}

For virus infection, $6 \times 10^{5}$ cells were seeded in 6-well plates the day before. pLKO.1 lentiviral vectors expressing small hairpin (sh) RNAs targeting DAXX (Sh-DAXX_H2410: and Sh-DAXX_2503) or a nontargeting shRNA control (SHCOO2) were purchased from Sigma-Aldrich. Lentiviral production and transduction were done as described in Tschan et al. (2003). Transduced cells were selected by puromycin treatment $(1.5 \mu \mathrm{g} / \mathrm{mL}$, Invitrogen). For micronuclei counting, cells were seeded in 24-well plates on coverslips coated with FBS, $1 \times 10^{4}$ cells per well. Cells were fixed with $4 \%$ paraformaldehyde for $30 \mathrm{~min}$ at room temperature. DAPI was used to stain nuclei. Micronuclei were counted under a fluorescent microscope (Axiophot2, Zeiss).

\section{Viability and apoptosis assays}

For MTT assay, cells were seeded into 96-well plates at the density of 16,000 cells per well. After $24 \mathrm{~h}$, medium was replaced by $100 \mu \mathrm{Ll}$ medium with $10 \%$ MTT $(0.5 \mathrm{mg} / \mathrm{mL}$ final concentration) (Sigma-Aldrich) and incubated for $1 \mathrm{~h}$ at $37^{\circ} \mathrm{C}$. After incubation, the medium was removed and $200 \mu \mathrm{L}$ of DMSO (Sigma-Aldrich) and $25 \mu \mathrm{L}$ of Sorensen solution ( $0.1 \mathrm{M}$ Glycine, $0.1 \mathrm{M} \mathrm{NaCl}$ and $\mathrm{pH} 10.5$ in water) were added into each well. Absorbance at $570 \mathrm{~nm}$ was determined on a spectrophotometer (Tecan).

\section{Cell cycle analysis with FACS}

Briefly, cells were seeded $5 \times 10^{5}$ cells in duplicates or triplicates in 6-well plates. The day after, cells were treated with Nocodazole. After $16 \mathrm{~h}$ of treatment, cells were fixed in ice-cold EtOH overnight. PI staining was performed directly before measurement on BD-FACS LSR SorpII using BD FACSDiva 6.1 software (BD Bioscience, Heidelberg, Germany). Results were analyzed using FlowJo v9.8 software (TreeStar, Ashland, OR, USA).

\section{Protein extraction and Western blot analysis}

For Western blotting, cells were cultivated in 6-well plates and lysate in urea buffer. Forty ng proteins were loaded on 4-20\% Mini-PROTEAN TGX Stain-Free Gel (Bio-Rad). Transfer was performed using transfer turbo blot system (Bio-Rad). Primary monoclonal antibodies rabbit antiDAXX (clone 25C12, 1:1000 in 5\% BSA, Cell signaling) and mouse anti-human $\alpha$-tubulin (clone B-5-1-2, 1:1000 in 5\% BSA, Sigma) and GAPDH (clone 6c5, 1:1000 in 5\% skimmed milk, Merk Millpore) were used. As secondary antibodies, goat anti-rabbit DyLight 650 conjugate (LabForce, Muttenz, Switzerland) and goat anti-mouse DyLight 550 conjugate (LabForce) both diluted 1:500 in $5 \%$ milk were used. Signal was detected with ChemiDoc MP System (Bio-Rad) and analyzed using ImageJ (freeware, $1.48 v$, Bethesda, USA). Protein levels were normalized to $\alpha$-tubulin, GAPDH or total protein.

\section{Statistics}

Statistical analysis was performed using GraphPad prism 5 software. Statistical differences were calculated using unpaired two-tailed Student's $t$ test. The $\chi^{2}$ test or the Fisher exact test was used to calculate contingency tables. $P$ values less than 0.05 were considered statistically significant.

Published by Bioscientifica Ltd 


\section{IHC 5-methylcytidine}
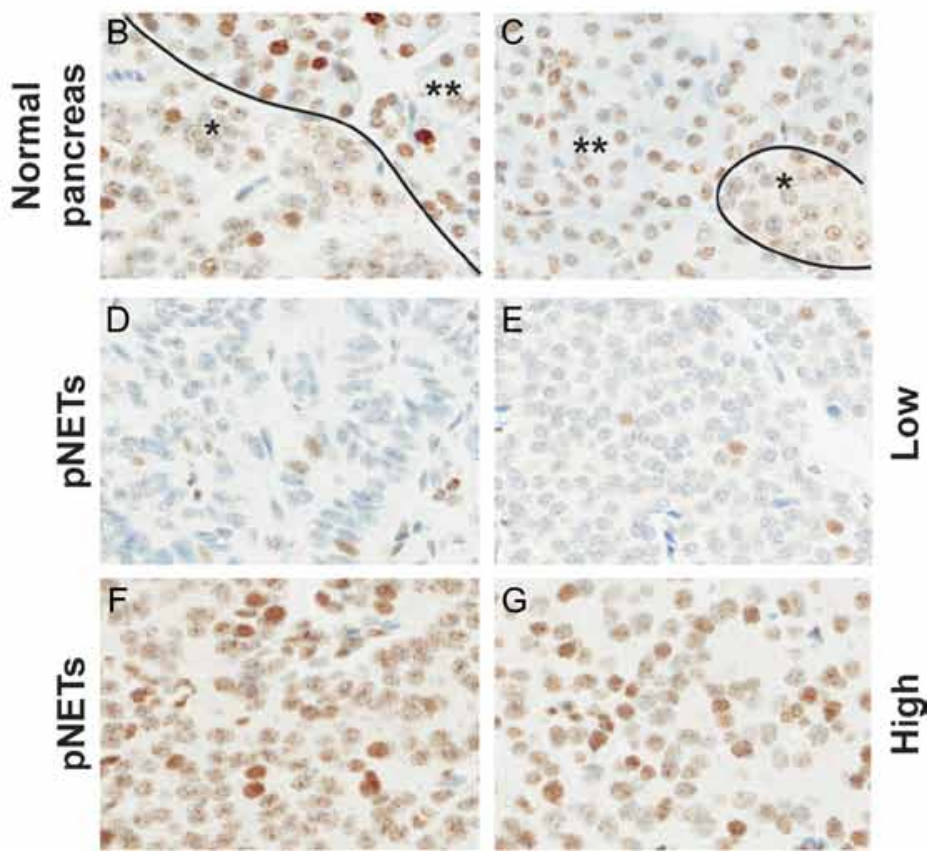

Figure 1

Global methylation in PanNETs. (A) Global methylation levels of DNA isolated from human PanNETs using EpiSeeker methylated DNA Quantification Kit (Abcam). Highly methylated DNA and unmethylated DNA were used as controls. DAXX/ATRX-/ALT+/CIN+ tumors showed hypo-methylation compared to DAXX/ATRX+/ALT-/CIN- ones $(P<0.01 * *)$. (B-G) IHC with anti-5-methylcytidine antibody (Millipore) on human tissues. (B and C) Normal pancreas: ${ }^{*}$ ) pancreatic islets and (**) exocrine pancreas. (D and E) Two PanNETs samples showing low 5-methlycytidine expression and (F and G) two PanNET samples showing high 5-methylcytidine expression.

\section{Results}

\section{Ex vivo analysis of human PanNET samples}

DAXX- and/or ATRX-negative PanNETs are hypomethylated To first assess whether DAXX/ATRX loss had an impact on the DNA methylation pattern, we evaluated global DNA methylation on 10 PanNET samples. DNA extracted from 5 PanNET samples positive for DAXX/ ATRX IHC staining, negative for ALT activation and CIN negative (DAXX/ATRX+/ALT-/CIN-) and from 5 PanNET samples negative for DAXX/ATRX staining, positive for ALT activation and CIN positive (DAXX/ATRX-/ALT+/ $\mathrm{CIN+)}$ were analyzed for global DNA methylation with the colorimetric EpiSeeker assay (methylated DNA Quantification Kit, Abcam). As shown in Figure 1A, DAXX/ATRX-/ALT+/CIN+ tumors exhibit a significantly lower level of DNA methylation compared to those of DAXX/ATRX+/ALT-/CIN- ones $\left(P<0.01^{* *}\right)$, which is comparable to the unmethylated control. To confirm our findings in a larger setting and with an additional method, we stained 2 TMAs, composed as described in the Materials and methods section and in Marinoni et al. (2014) with an anti-5-methylcytidine antibody (Eurogentec) that specifically recognizes the methylated base and does not cross-react with the unmethylated one. We obtained results on 153 samples; the characteristic of these samples is reported in Table 1.

We observed that strong 5-methylcytidine staining associates with DAXX- and ATRX-proficient tumors, whereas weak signal correlates with DAXX/ATRXdeficient ones $\left(P<0.05^{*}\right)$ (Fig. 1B, C, D, E, F and G and Table 2), suggesting that DAXX/ATRX-deficient tumor shows global hypo-methylation compared to the positive ones. Normal islets showed a strong 5-methylcytidine staining (Fig. 1B and C).

Table 2 Correlation between DAXX/ATRX expression and 5-methylcytidine staining in human PanNET tissues.

\begin{tabular}{|c|c|c|c|}
\hline & $\begin{array}{c}\text { Low } \\
\text { 5-methylcytidine }\end{array}$ & $\begin{array}{c}\text { High } \\
\text { 5-methylcytidine }\end{array}$ & $P$ value \\
\hline DAXX/ATRX- & 28 & 15 & $0.019 *$ \\
\hline DAXX/ATRX+ & 28 & 39 & \\
\hline DAXX- & 17 & 6 & $0.020 *$ \\
\hline DAXX+ & 44 & 52 & \\
\hline ATRX- & 15 & 13 & 0.517 \\
\hline ATRX+ & 40 & 48 & \\
\hline ALT- & 25 & 28 & 0.338 \\
\hline ALT+ & 21 & 15 & \\
\hline
\end{tabular}

Published by Bioscientifica Ltd. 
To examine the DNA methylation level of noncoding regions, we evaluated the (long interspersed element) LINE1 methylation status as a marker of global DNA methylation in 55 PanNET and 11 non-neoplastic pancreatic specimens, by pyrosequencing (Qiagen). We observed that LINE1 is hypo-methylated in PanNETs compared to normal pancreas $\left(P<0.001^{* * *}\right)$ (Fig. 2A) as previously reported (Choi et al. 2007, Stefanoli et al. 2014). LINE1 hypo-methylation indeed associates with CIN $\left(P<0.05^{*}\right)$ (Fig. 2B), as we hypothesized. However, no differences in LINE1 methylation were observed between DAXX/ATRX-positive and -negative tumors, or between ALT-positive and -negative tumors (Fig. 2C, D and E). To assess if MEN1 mutations influence LINE1 methylation levels, we sequenced MEN1 gene in 26 samples. Eleven samples resulted to be mutated. The mutations found are summarized in Supplementary Table 1. No difference in LINE1 methylation status was observed between mutant and wild-type tumors (Fig. 2F). Interestingly, LINE1 hypomethylation predicts for shorter disease-free survival $\left(P<0.05^{*}\right)$, (Supplementary Fig. 3) in agreement with the results reported by Stefanoli et al. (2014), whereas no differences in survival are detectable in tumors positive and negative for 5-methylcytidine staining (data not shown).

We performed quantitative methylation-specific PCR to assess whether LINE1 hypo-methylation or DAXX/ ATRX loss correlate with hyper-methylation of MGMT promoter, due to its clinical relevance. We found 8 samples with MGMT promoter hyper-methylated and 17 samples where MGMT promoter was unmethylated. No correlation between LINE1 hypo-methylation or DAXX/ATRX loss and MGMT methylation level was found (Supplementary Fig. 4), suggesting two independent mechanisms for the two events, as previously proposed (Stefanoli et al. 2014).

\section{In vitro upon DAXX knock-down}

Induction of genomic instability and G1 arrest To assess if DAXX loss is sufficient to induce genomic instability, we knocked down DAXX in two PanNET cell lines, QGP-1 and BON-1 by lentiviral transduction of SH-RNAs. DAXX knock-down was evaluated by Western blotting 9, 17 and 24 days after lentiviral infection. Two
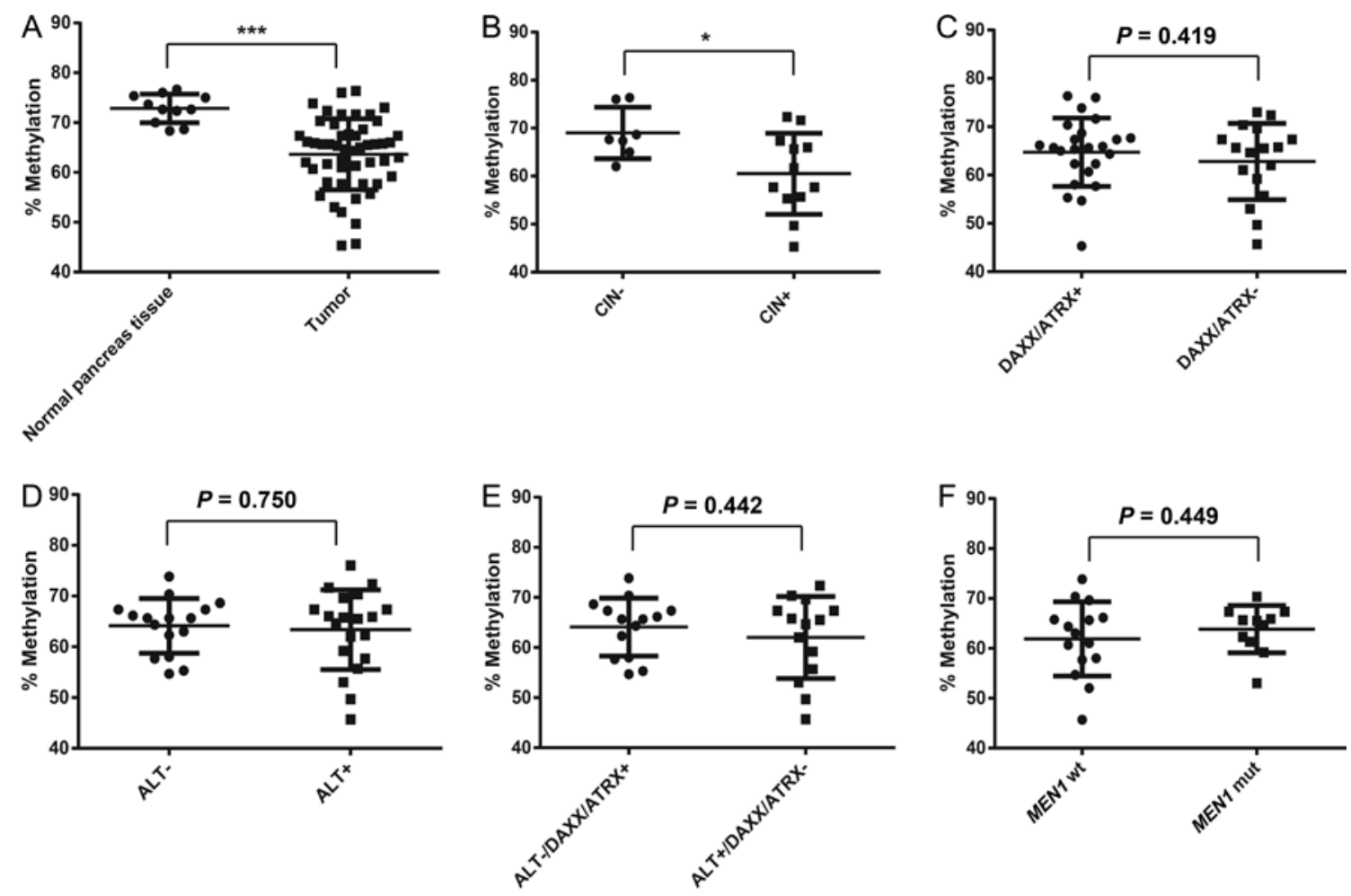

Figure 2

LINE-1 methylation in PanNETs. (A) PanNET samples showed a significant lower level of methylation compared to normal surrounding tissues $(P<0.001 *)$. (B) PanNETs with high CIN showed a lower level of LINE1 methylation $(P<0.05)$ compared to tumors with no CIN. (C and D) DAXX/ATRX- tumors and ALT+ tumors do not show difference in LINE1 methylation compared to, respectively, DAXX/ATRX+ and ALT- tumors. (E) Tumors that are both DAXX/ATRX- and ALT+ did not show difference in LINE1 methylation level compared to tumors, which are DAXX/ATRX+ and ALT-, (F) MEN1 mutated and wild-type PanNET do not show differences in LINE1 methylation levels. 
A

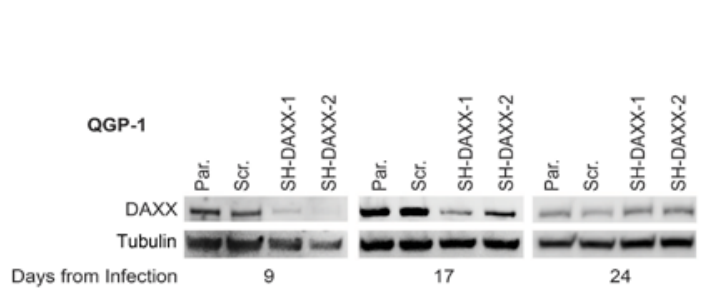

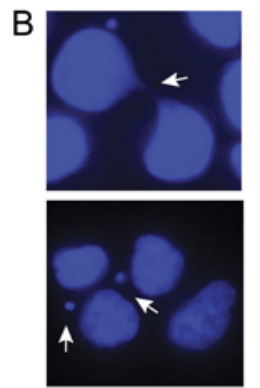

C

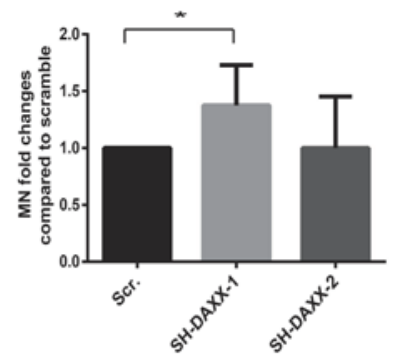

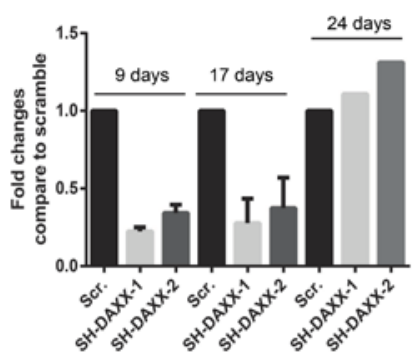

D

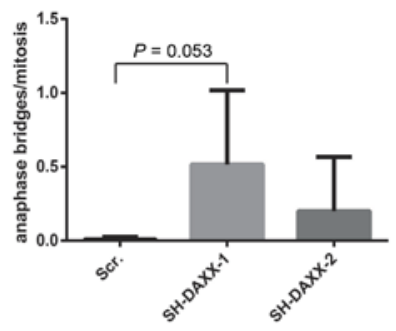

16 days from infection

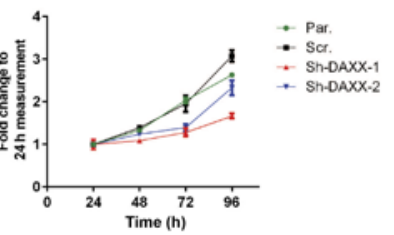

E

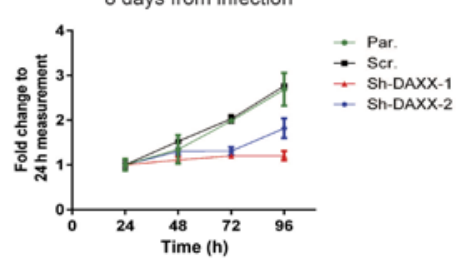

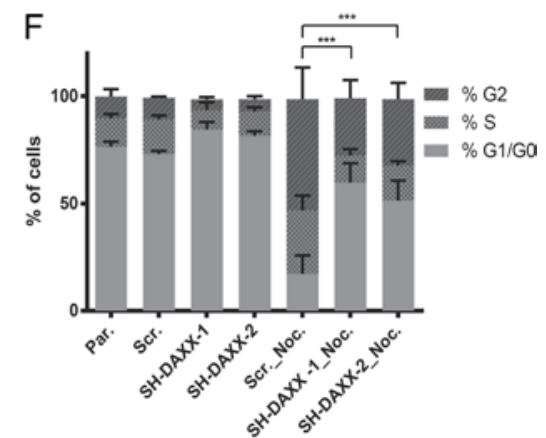

Figure 3

DAXX knock-down in QGP-1 cell lines. (A) Western blotting showing DAXX knock-down after 9, 17 and 24 days upon infection with two different plasmids SH-DAXX-1 and SH-DAXX-2 in QGP-1. Parental (Par.) cells and cells transduced with scramble vector (Scr.) were used as controls. DAXX expression comes back to normal level after 24 days from the infection. Quantification of the knock-down after 9 and 17 days from infection was based on three different experiments. (B) DAPI staining of QGP-1 cells after DAXX knock-down; arrows indicate an anaphase bridge and micronuclei. (C) Fold changes of the number of micronuclei (MN) per cells compare to scramble. (D) Number of anaphase bridges compare to mitosis. SH-DAXX-1 and SH-DAXX-2 cells showed an increased on micronuclei and anaphase bridges compared to controls. (E) Graphic representation of MTT viability assays after 8 days and after 16 days from infection. DAXX knock-down impairs cell viability. After 16 days, when DAXX expression is restored to normal levels, the cells proliferate as the controls, suggesting a direct effect of DAXX on cell viability. (F) FACS analysis of QGP1 cells after DAXX knock-down. Cells knocked down for DAXX showed an increased in the percentage of cells in $\mathrm{G} 1 / \mathrm{G} 0$ compared to controls. The results are based on at least three repetitions.

different DAXX-specific vectors (1 and 2) and scramble control were used in both cell lines. DAXX expression is highly reduced after 9 days from infections in both cell lines (Fig. 3A and Supplementary Fig. 5A). After 17 days, DAXX expression is downregulated to a lesser extent, whereas both cell lines re-express DAXX after 24 days. Therefore, all experiments were performed within the first 10 days after infection. Of note, the knock-down efficiency was higher with vector SH-DAXX-1 than SH-DAXX-2; consistently, we observed a stronger phenotype on the cells transduced with the first vector.

Interestingly, we observed that in QGP-1 cells, DAXX knock-down induced an increased number of micronuclei formation and anaphase bridges, whereas no micronuclei formation was observed in BON-1 cells. DAXX knockdown QGP-1 cells showed an increased number of micronuclei formation compared to scramble (Fig. 3B, $\mathrm{C}$ and $\mathrm{D})$. This result suggests an increased genomic instability induced by DAXX loss in QGP-1 cells.

DAXX knock-down in both QGP-1 and BON-1 does not induce ALT activation as detected by telomere FISH in vitro (data not shown), similar to what has been shown by others in ATRX knock-down cells (Napier et al. 2015).

DAXX knock-down reduced cell viability, measured by MTT assay (Fig. 3E and Supplementary Fig. 5B), indicating that DAXX does not have a classical tumor suppressor gene function. No significant increase in apoptosis was observed in DAXX knock-down samples

Published by Bioscientifica Ltd 
compared to that in the scramble control (Supplementary Fig. 6). FACS analysis revealed that DAXX knock-down induces $\mathrm{G} 1$ arrest in both BON-1 cells and QGP-1 (Fig. 3F and Supplementary Fig. 7).

\section{Short-time DAXX knock-down does not affect LINE1 and global methylation}

As in human samples we observed that DAXX and or ATRX loss correlates with DNA hypo-methylation, we wanted to investigate whether DAXX knock-down impairs DNA methylation in vitro as well. No difference in LINE1 methylation as well as global methylation was observed after 10 days of DAXX knock-down in both BON-1 and QGP-1 cells (Supplementary Fig. 8 and data not shown). Similarly, no difference in MGMT promoter methylation was observed upon DAXX knock-down (data not shown).

\section{Discussion}

The sequence of the events and the mechanism by which DAXX and or ATRX loss induces ALT and CIN are still unknown. Here, we provide evidence that epigenetic mechanisms could be involved in this process. In detail, we showed by immunohistochemistry and biochemical methods that DAXX- and/or ATRX-negative PanNETs are globally hypo-methylated. Hypo-methylation of repeated sequences has been described in several cancers in correlation with progressive increase of the grade of malignancy (Ehrlich 2002). Long interspersed nuclear elements (LINE1) are the most abundant mobile DNAs in the human genome, and hypo-methylation of these sequences has been shown in different types of cancer including PanNETs (Choi et al. 2007, Stefanoli et al. 2014). Indeed, we could confirm that LINE1 is hypo-methylated in PanNET compared to that in normal pancreas. Here, we additionally showed that PanNETs showing CIN have a lower level of LINE1 methylation than chromosomally stable PanNET. We previously showed that DAXX and or ATRX loss in PanNETs correlates with CIN (Marinoni et al. 2014). We hypothesize that DAXX and ATRX loss in PanNET cells result in a decreased DNA methylation, thus promoting CIN. Indeed, we found that DAXX/ATRXnegative PanNET shows lower level of global methylation assessed by IHC and by a colorimetric assay.

However, we could not find a correlation between DAXX and or ATRX loss, ALT activation and LINE1 methylation. LINE1 methylation could therefore be mediated by other unknown events. On the other hand, a multi-step model in which DAXX and ATRX loss progressively impairs DNA methylation and chromatin structure would also explain this discrepancy. Indeed, our cell line results could point into this direction: Shorttime DAXX knock-down did not induce any changes in global or LINE1 DNA methylation. Either DAXX knockdown is not sufficient to induce global hypo-methylation without other events or it is instead a matter of time and additional cell cycles are needed.

Impairment of DNA methylation, particularly at telomeres, has been described in glioblastoma with ALT activation as well as in astrocytoma with low ATRX expression (Sturm et al. 2012, Cai et al. 2015). Recently, it has been shown that DAXX-deficient PanNET showed higher methylation variation compared to ATRX-negative ones (Pipinikas et al. 2015). However, this report did not focus on global methylation levels, which could largely be influenced by non-coding sequences, such as LINE1 and telomeric regions.

A reduction of methylation in the subtelomeric regions, allowing DNA recombination, might also promote ALT activation (Gonzalo et al. 2006). Upon DAXX knock-down in PanNET cell lines, we did not observe ALT activation, in keeping with other findings showing that DAXX and ATRX loss does not induce ALT activation in vitro in telomerase-positive cells (Napier et al. 2015). In QGP-1 cells, we observed a higher number of micronuclei upon DAXX knock-down compared to control, which might be a sign of increased genomic instability already at this short time point and without ALT phenotype and impairment in DNA methylation. However, the small percentage, of cells showing micronuclei formation upon DAXX knockout might not be sufficient to detect DNA methylation changes when this is assessed in the whole cell population.

The phenotype of DAXX knock-down cells has confirmed the non-conventional tumor suppressor role. DAXX silencing in vitro in BON-1 and QGP-1 cells induced G1/G0 cell cycle arrest. Similarly, ATRX knockdown in other tumor models reduced cell proliferation and induced genomic instability (Huh et al. 2012, Lovejoy et al. 2012, Cai et al. 2015, Huh et al. 2016). It is important to point out that BON-1 and QGP-1 cells are mainly a model for G3 PanNECs with mutations in genes, which are usually not altered in G1-G2 PanNETs (i.e. TP53) (Vandamme et al. 2015). In this context, DAXX knock-down may influence the cell cycle regulation and genomic instability differently than that in the slow progressive tumors with a different mutational spectrum. 
Additionally, the difference in the mutations background between QGP-1 and BON-1 may explain the different results in the micronuclei formation. Recently, it has been shown that Daxx knock-down in a rat insulinoma cell line increased cell proliferation, suggesting that this model might be more appropriate for Daxx functional studies (Feng et al. 2016). However, rodent cells usually show minor and different genomic instability patterns compared to human cells and they rarely activate ALT mechanism for telomere lengthening, even in the absence of telomerase (Argilla et al. 2004, Hermsen et al. 2015). This suggests that even this model harbors some limitation for the study of the impact of DAXX loss on genomic instability, ALT activation and DNA methylation, which was the main focus of our work.

Our finding very likely only show a part of a more complex situation as other factors than DAXX, and ATRX mutation can influence the epigenetic status. DAXX and/or ATRX loss is frequently accompanied by MEN1 mutations, and this possibly contributes to the epigenetic status. MEN1 is mutated in almost 44\% of sporadic PanNETs (Jiao et al. 2011). MEN1 gene, encodes the transcription factor menin, which recruits the H3K4me3 histone methyltransferase mixed lineage leukemia (MLL1) complex that plays an essential role in chromatin remodeling and gene expression (Agarwal et al. 1999, Agarwal \& Jothi 2012, Yang et al. 2013). However, MEN1 mutations in PanNETs are not associated with CIN or with ALT phenotype. In agreement with a model of epigenetically induced CIN, our results showed no correlation between LINE1 methylation level and MEN1 mutation status. Similarly, we did not detect any correlation between global methylation assessed by IHC and MEN1 mutations (data not shown).

In conclusion, our data provide evidence that DAXX and ATRX loss has an impact on global DNA methylation of PanNETs cells, which seem to be involved in chromosomal instability. This could enable PanNET to acquire clonal heterogeneity leading to a selection of more aggressive clones.

\section{Supplementary data}

This is linked to the online version of the paper at http://dx.doi.org/10.1530/ ERC-16-0554.

\section{Declaration of interest}

The authors declare that there is no conflict of interest that could be perceived as prejudicing the impartiality of the research reported.

\section{Funding}

The study was supported by the Swiss National Science foundation (SNF Grant No. 310030_144236 to Aurel Perren and SNF-Marie Heim Vögtlin (PMPDP3_164484/1) to Ilaria Marinoni) and by Tumor Forschung Bern to Ilaria Marinoni.

\section{Author contribution statement}

Ilaria Marinoni: experimental design, supervision and execution of in vitro and ex-vivo experiments, manuscript writing. Astrid Wiederkeher: execution of the in vitro experiments. Tabea Wiedmer: execution of the FACS analysis and critical reading of the manuscript. Sophia Pantasis: LINE1 pyrosequencing. Rasmus Frank: LINE1 pyrosequencing and MGMT methylation. Annunziata Di Domenico: DNA extraction and samples collection. Erik Vassella: MEN1 sequencing and MGMT methylation analysis. Anja Schmitt: Acquisition of clinical data and critical reading of the manuscript. Aurel Perren: experimental design, data acquisition of exvivo experiments, morphological analysis and manuscript writing.

\section{Acknowledgements}

The authors would like to thank Prof. Mario Tschan for the Lentivirus plasmids and for the support with all the Lentivirus experiments. Cornelia Schlup for the support with NGS and pyrosequencing. The Translation Research Unit and in particular Dr Jose Galvanez for cutting the slides and the Immunohistochemistry. Tissues were provided with support of the Tissue Bank Bern (TBB).

\section{References}

Agarwal SK \& Jothi R 2012 Genome-wide characterization of menindependent H3K4me3 reveals a specific role for menin in the regulation of genes implicated in MEN1-like tumors. PLOS ONE 7 e37952. (doi:10.1371/journal.pone.0037952)

Agarwal SK, Guru SC, Heppner C, Erdos MR, Collins RM, Park SY, Saggar S, Chandrasekharappa SC, Collins FS, Spiegel AM, et al. 1999 Menin interacts with the AP1 transcription factor JunD and represses JunD-activated transcription. Cell 96 143-152. (doi:10.1016/S00928674(00)80967-8)

Argilla D, Chin K, Singh M, Hodgson JG, Bosenberg M, de Solorzano CO, Lockett S, DePinho RA, Gray J \& Hanahan D 2004 Absence of telomerase and shortened telomeres have minimal effects on skin and pancreatic carcinogenesis elicited by viral oncogenes. Cancer Cell 6 373-385. (doi:10.1016/j.ccr.2004.08.032)

Cai J, Chen J, Zhang W, Yang P, Zhang C, Li M, Yao K, Wang H, Li Q, Jiang C, et al. 2015 Loss of ATRX, associated with DNA methylation pattern of chromosome end, impacted biological behaviors of astrocytic tumors. Oncotarget 6 18105-18115. (doi:10.18632/oncotarget.3906)

Choi IS, Estecio MR, Nagano Y, Kim do H, White JA, Yao JC, Issa JP \& Rashid A 2007 Hypomethylation of LINE-1 and Alu in welldifferentiated neuroendocrine tumors (pancreatic endocrine tumors and carcinoid tumors). Modern Pathology 20 802-810. (doi:10.1038/ modpathol.3800825)

Das PM \& Singal R 2004 DNA methylation and cancer. Journal of Clinical Oncology 22 4632-4642. (doi:10.1200/JCO.2004.07.151)

Ehrlich M 2002 DNA methylation in cancer: too much, but also too little. Oncogene 21 5400-5413. (doi:10.1038/sj.onc.1205651)

Feng Z, Wang L, Sun Y, Jiang Z, Domsic J, An C, Xing B, Tian J, Liu X, Metz DC, et al. 2016 Menin and Daxx interact to control neuroendocrine tumors via epigenetic regulation of membrane metallo-endopeptidase. Cancer Research 77 401-411. (doi:10.1158/0008-5472.CAN-16-1567) 
Gonzalo S, Jaco I, Fraga MF, Chen T, Li E, Esteller M \& Blasco MA 2006 DNA methyltransferases control telomere length and telomere recombination in mammalian cells. Nature Cell Biology 8 416-424. (doi:10.1038/ncb1386)

Heaphy CM, de Wilde RF, Jiao Y, Klein AP, Edil BH, Shi C, Bettegowda C, Rodriguez FJ, Eberhart CG, Hebbar S, et al. 2011 Altered telomeres in tumors with ATRX and DAXX mutations. Science 333425 (doi:10.1126/science.1207313)

Hermsen R, Toonen P, Kuijk E, Youssef SA, Kuiper R, van Heesch S, de Bruin A, Cuppen E \& Simonis M 2015 Lack of major genome instability in tumors of p53 null rats. PLOS ONE 10 e0122066. (doi:10.1371/journal.pone.0122066)

House MG, Herman JG, Guo MZ, Hooker CM, Schulick RD, Lillemoe KD, Cameron JL, Hruban RH, Maitra A \& Yeo CJ 2003 Aberrant hypermethylation of tumor suppressor genes in pancreatic endocrine neoplasms. Annals of Surgery 238 423-431; discussion 431-422. (doi:10.1097/01.sla.0000086659.49569.9e)

Huh MS, Price O"Dea T, Ouazia D, McKay BC, Parise G, Parks RJ, Rudnicki MA \& Picketts DJ 2012 Compromised genomic integrity impedes muscle growth after Atrx inactivation. Journal of Clinical Investigation 122 4412-4423. (doi:10.1172/JCI63765)

Huh MS, Ivanochko D, Hashem LE, Curtin M, Delorme M, Goodall E, Yan K \& Picketts DJ 2016 Stalled replication forks within heterochromatin require ATRX for protection. Cell Death \& Disease 7 e2220. (doi:10.1038/cddis.2016.121)

Jiao Y, Shi C, Edil BH, de Wilde RF, Klimstra DS, Maitra A, Schulick RD, Tang LH, Wolfgang CL, Choti MA, et al. 2011 DAXX/ATRX, MEN1, and mTOR pathway genes are frequently altered in pancreatic neuroendocrine tumors. Science 331 1199-1203. (doi:10.1126/ science.1200609)

Lovejoy CA, Li W, Reisenweber S, Thongthip S, Bruno J, de Lange T, De S, Petrini JH, Sung PA, Jasin M, et al. 2012 Loss of ATRX, genome instability, and an altered DNA damage response are hallmarks of the alternative lengthening of telomeres pathway. PLoS Genetics $\mathbf{8}$ e1002772. (doi:10.1371/journal.pgen.1002772)

Malpeli G, Amato E, Dandrea M, Fumagalli C, Debattisti V, Boninsegna L, Pelosi G, Falconi M \& Scarpa A 2011 Methylationassociated down-regulation of RASSF1A and up-regulation of RASSF1C in pancreatic endocrine tumors. BMC Cancer 11351. (doi:10.1186/1471-2407-11-351)

Marinoni I, Kurrer AS, Vassella E, Dettmer M, Rudolph T, Banz V, Hunger F, Pasquinelli S, Speel EJ \& Perren A 2014 Loss of DAXX and ATRX are associated with chromosome instability and reduced survival of patients with pancreatic neuroendocrine tumors. Gastroenterology 146 453-460 e455. (doi:10.1053/j. gastro.2013.10.020)

Napier CE, Huschtscha LI, Harvey A, Bower K, Noble JR, Hendrickson EA \& Reddel RR 2015 ATRX represses alternative lengthening of telomeres. Oncotarget 6 16543-16558. (doi:10.18632/oncotarget.3846)

Noh KM, Allis CD \& Li H 2016 Reading between the Lines: 'ADD'-ing Histone and DNA methylation marks toward a new epigenetic
'Sum'. ACS Chemical Biology 11 554-563. (doi:10.1021/ acschembio.5b00830)

Pipinikas CP, Dibra H, Karpathakis A, Feber A, Novelli M, Oukrif D, Fusai G, Valente R, Caplin M, Meyer T, et al. 2015 Epigenetic dysregulation and poorer prognosis in DAXX-deficient pancreatic neuroendocrine tumours. Endocrine-Related Cancer 22 L13-L18. (doi:10.1530/ERC-15-0108)

Puto LA \& Reed JC 2008 Daxx represses RelB target promoters via DNA methyltransferase recruitment and DNA hypermethylation. Genes \& Development 22 998-1010. (doi:10.1101/gad.1632208)

Schmitt AM, Schmid S, Rudolph T, Anlauf M, Prinz C, Kloppel G, Moch H, Heitz PU, Komminoth P \& Perren A 2009 VHL inactivation is an important pathway for the development of malignant sporadic pancreatic endocrine tumors. Endocrine-Related Cancer 16 1219-1227. (doi:10.1677/ERC-08-0297)

Schmitt AM, Pavel M, Rudolph T, Dawson H, Blank A, Komminoth P, Vassella E \& Perren A 2014 Prognostic and predictive roles of MGMT protein expression and promoter methylation in sporadic pancreatic neuroendocrine neoplasms. Neuroendocrinology 100 35-44. (doi:10.1159/000365514)

Stefanoli M, La Rosa S, Sahnane N, Romualdi C, Pastorino R, Marando A, Capella C, Sessa F \& Furlan D 2014 Prognostic relevance of aberrant DNA methylation in G1 and G2 pancreatic neuroendocrine tumors. Neuroendocrinology 100 26-34. (doi:10.1159/000365449)

Sturm D, Witt H, Hovestadt V, Khuong-Quang DA, Jones DT, Konermann C, Pfaff E, Tonjes M, Sill M, Bender S, et al. 2012 Hotspot mutations in H3F3A and IDH1 define distinct epigenetic and biological subgroups of glioblastoma. Cancer Cell 22 425-437. (doi:10.1016/j.ccr.2012.08.024)

Tschan MP, Fischer KM, Fung VS, Pirnia F, Borner MM, Fey MF, Tobler A \& Torbett BE 2003 Alternative splicing of the human cyclin D-binding Myb-like protein (hDMP1) yields a truncated protein isoform that alters macrophage differentiation patterns. Journal of Biological Chemistry 278 42750-42760. (doi:10.1074/jbc. M307067200)

Vandamme T, Peeters M, Dogan F, Pauwels P, Van Assche E, Beyens M, Mortier G, Vandeweyer G, de Herder W, Van Camp G, et al. 2015 Whole-exome characterization of pancreatic neuroendocrine tumor cell lines BON-1 and QGP-1. Journal of Molecular Endocrinology 54 137-147. (doi:10.1530/JME-14-0304)

Vassella E, Vajtai I, Bandi N, Arnold M, Kocher V \& Mariani L 2011 Primer extension based quantitative polymerase chain reaction reveals consistent differences in the methylation status of the MGMT promoter in diffusely infiltrating gliomas (WHO grade II-IV) of adults. Journal of Neuro-Oncology 104 293-303. (doi:10.1007/ s11060-010-0490-4)

Yang YJ, Song TY, Park J, Lee J, Lim J, Jang H, Kim YN, Yang JH, Song Y, Choi A, et al. 2013 Menin mediates epigenetic regulation via histone H3 lysine 9 methylation. Cell Death \& Disease 4 e583. (doi:10.1038/ cddis.2013.98)

Received in final form 23 December 2016

Accepted 23 January 2017

Accepted Preprint published online 23 January 2017
๑ 2017 Society for Endocrinology Printed in Great Britain
Published by Bioscientifica Ltd. 